\title{
RNA-seq analysis of differential gene expression in liver from lactating dairy cows divergent in negative energy balance
}

\author{
Matthew McCabe ${ }^{1}$, Sinéad Waters ${ }^{1}$, Dermot Morris², David Kenny ${ }^{2}$, David Lynn ${ }^{1}$ and Chris Creevey ${ }^{1 *}$
}

\begin{abstract}
Background: The liver is central to most economically important metabolic processes in cattle. However, the changes in expression of genes that drive these processes remain incompletely characterised. RNA-seq is the new gold standard for whole transcriptome analysis but so far there are no reports of its application to analysis of differential gene expression in cattle liver. We used RNA-seq to study differences in expression profiles of hepatic genes and their associated pathways in individual cattle in either mild negative energy balance (MNEB) or severe negative energy balance (SNEB). NEB is an imbalance between energy intake and energy requirements for lactation and body maintenance. This aberrant metabolic state affects high-yielding dairy cows after calving and is of considerable economic importance because of its negative impact on fertility and health in dairy herds. Analysis of changes in hepatic gene expression in SNEB animals will increase our understanding of NEB and contribute to the development of strategies to circumvent it.

Results: RNA-seq analysis was carried out on total RNA from liver from early post partum Holstein Friesian cows in MNEB $(n=5)$ and SNEB $(n=6) .12,833$ genes were deemed to be expressed ( $>4$ reads per gene per animal), 413 of which were shown to be statistically significantly differentially expressed (SDE) at a false discovery rate (FDR) of $0.1 \%$ and 200 of which were SDE (FDR of $0.1 \%$ ) with a $\geq 2$-fold change between MNEB and SNEB animals. GOseq/ KEGG pathway analysis showed that SDE genes with $\geq 2$ - fold change were associated $(P<0.05)$ with 9 KEGG pathways. Seven of these pathways were related to fatty acid metabolism and unexpectedly included 'Steroid hormone biosynthesis', a process which mainly occurs in the reproductive organs rather than the liver.

Conclusions: RNA-seq analysis showed that the major changes at the level of transcription in the liver of SNEB cows were related to fat metabolism. 'Steroid hormone biosynthesis', a process that normally occurs in reproductive tissue, was significantly associated with changes in gene expression in the liver of SNEB cows. Changes in gene expression were found in this pathway that have not been previously been identified in SNEB cows.
\end{abstract}

Keywords: RNA-seq, Negative energy balance, Bovine, Liver

\section{Background}

Selective breeding for high milk yield in dairy cows has led to breeds in which the nutritional demands of the very high lactation rates following calving are in excess of that which the animal can metabolise from ingested feed [1]. This aberrant physiological state is known as negative energy balance (NEB) and is of particular

\footnotetext{
* Correspondence: chris.creevey@teagasc.ie

${ }^{1}$ Animal and Bioscience Research Department, Animal and Grassland Research and Innovation Centre, Teagasc, Grange, Dunsany, Co. Meath, Ireland

Full list of author information is available at the end of the article
}

economic importance in the dairy industry because of its negative impact on health and fertility [1].

The principal physiological response of animals to NEB is to try and maintain homeostasis by mobilising body fat (and protein) reserves. This results in the release of non-esterified fatty acids (NEFAs) from adipose. NEFAs are transported primarily to the liver where they are either fully oxidized to $\mathrm{CO}_{2}$, or converted to ketone bodies such as beta-hydroxybutyrate (BHB), or esterified into triacylglycerides (TAG) either for delivery into blood as very low density lipoproteins proteins (VLDL) or storage as cytosolic lipid droplets [2]. As with all

\section{Biomed Central}


ruminants, dairy cattle have very low rates of VLDL synthesis and secretion [3] and are therefore susceptible to accumulation of high levels of TAG in the liver cells (referred to as fatty liver or lipidosis) and high concentrations of ketone bodies in the blood (ketosis) [4] both of which are potentially detrimental to the health of the animals [5]. Although there are strategies to prevent and treat NEB, such as management of diet and inclusion of dietary supplements [6], [7] NEB and ketosis continue to have a significant negative economic impact in dairy cattle. This has led to an effort to understand NEB at the level of genes and their expression [8].

As with the majority of metabolic states, changes in gene expression in the liver are central to NEB. There have been several studies describing such changes at the level of transcription in the liver of NEB cows which have employed qPCR [9] and bovine microarrays $[10,11]$. However, microarray analyses rely on existing genome annotations and require design of a specialised array of probes based on information obtained from other methods such as sequencing in order to detect more complex regulations in gene expression such as alternative splicing [12]. Furthermore, the insensitivity of microarray platforms in detecting differential expression of certain metabolically important genes has been previously highlighted by our own group who found that qPCR was able to detect changes in hepatic gene expression SNEB cows which microarray analysis failed to detect [11]. This emphasises the need for a more sensitive technique for whole transcriptome analysis.

RNA-seq is a relatively new technique that can be used to analyse changes in gene expression across the entire transcriptome $[13,14]$, and is now being applied to a rapidly increasing number of organisms [12]. This technology has distinct advantages over microarrays including the sensitive detection of all expressed genes without the need to generate an array of probes based on known sequence, virtually no background noise and a much higher dynamic range. It has also led to the discovery of previously unidentified transcripts such as the recent discovery of enhancer RNA, a novel RNA species [15] and as the transcriptome is being sequenced, alternative splicing [16], single nucleotide polymorphisms (SNPs) [17], transcriptional fusions and chimeric transcripts [18] can be detected without the need to design an array of probes.

Unlike microarrays, the bioinformatic tools for the analysis RNA-seq data are still at the early stages of development $[19,20]$. Partly for this reason, there are few reports to date on the use of RNA-seq for identification of significantly differentially expressed (SDE) genes between physiologically different groups of animals and, so far, no such reports for cattle. The use of RNA-seq to identify differential gene expression in two pools of cattle embryos that were divergent for viability was recently reported [12]. However, as RNA from two pools of 20 embryos was analysed rather than individual embryos, the authors were unable to apply statistical analysis to their data to determine statistical significance of the differential gene expression.

Here we describe the use of DEseq [21] and GOseq [22] to identify SDE genes and associated over-represented biological KEGG pathways across the whole liver transcriptome of individual cattle. For this we compared RNA samples from liver of mild and severe NEB (MNEB and SNEB) cows. The objective of our study was to use RNA-seq to identify novel genes and their associated biological pathways which are important in SNEB in cattle.

\section{Results and discussion}

Library preparation and sequencing of polyA mRNA-seq libraries

Twelve polyA RNA-seq single read libraries that had been prepared from total RNA extracted from liver from 6 MNEB animals (libraries 1-6) and 6 SNEB animals (libraries 7-12) were run as 36 bp single reads on 21 lanes randomly distributed across 3 flowcells (Table 1). Several libraries were run on one or more lanes at the same or different concentrations to determine the effect of library concentration on cluster generation and to assess the consistency of sequence between different lanes and flow cells. For the 21 lanes, the average number of raw reads per lane was 13.66 million. Reads were aligned to the BCM4 genome assembly [23], using the ultrafast short read aligner, Bowtie (version 0.12.3) [24]. Following

Table 1 Summary of RNA-seq libraries and flow cells

\begin{tabular}{|c|c|c|c|c|c|}
\hline Lane & Flow cell A & Lane & Flow cell B & Lane & Flow cell $\mathrm{C}$ \\
\hline$\overline{1 A}$ & $\begin{array}{l}\text { library } 1 \\
(4.5 \mathrm{pM})\end{array}$ & $1 \mathrm{~B}$ & $\begin{array}{l}\text { library } 12 \\
(5.5 \mathrm{pM})\end{array}$ & $1 \mathrm{C}$ & $\begin{array}{l}\text { library } 2 \\
\text { (5.5 pM) }\end{array}$ \\
\hline $2 \mathrm{~A}$ & $\begin{array}{l}\text { library1 } \\
\text { (5.0 pM) }\end{array}$ & $2 B$ & $\begin{array}{l}\text { library } 6 \\
\text { (5.5 pM) }\end{array}$ & $2 C$ & $\begin{array}{l}\text { library } 3 \\
\text { (5.5 pM) }\end{array}$ \\
\hline $3 \mathrm{~A}$ & $\begin{array}{l}\text { library } 5 \\
\text { (5.5 pM) }\end{array}$ & $3 B$ & $\begin{array}{l}\text { library } 2 \\
\text { (5.5 pM) }\end{array}$ & $3 C$ & $\begin{array}{l}\text { library } 6 \\
\text { (5.5 pM) }\end{array}$ \\
\hline $4 \mathrm{~A}$ & $\begin{array}{l}\text { library5 } \\
\text { (6.0 pM) }\end{array}$ & $4 \mathrm{~B}$ & $\begin{array}{l}\text { library } 7 \\
(5.5 \mathrm{pM})\end{array}$ & $4 C$ & $\begin{array}{l}\text { library } 7 \\
\text { (5.5 pM) }\end{array}$ \\
\hline $5 \mathrm{~A}$ & $\begin{array}{l}\text { library } 10 \\
(4.0 \mathrm{pM})\end{array}$ & $5 B$ & $\begin{array}{l}\text { library } 9 \\
(5.5 \mathrm{pM})\end{array}$ & $5 C$ & PhiX \\
\hline $6 \mathrm{~A}$ & Phix & $6 \mathrm{~B}$ & PhiX & $6 C$ & $\begin{array}{l}\text { library } 8 \\
(5.5 \text { pM) }\end{array}$ \\
\hline $7 \mathrm{~A}$ & $\begin{array}{l}\text { library } 10 \\
(5.5 \mathrm{pM})\end{array}$ & $7 \mathrm{~B}$ & $\begin{array}{l}\text { library } 11 \\
(5.5 \mathrm{pM})\end{array}$ & $7 C$ & $\begin{array}{l}\text { library } 11 \\
(5.5 \mathrm{pM})\end{array}$ \\
\hline $8 \mathrm{~A}$ & $\begin{array}{l}\text { library } 10 \\
\text { (6.5 pM) }\end{array}$ & $8 B$ & $\begin{array}{l}\text { library } 4 \\
\text { (5.5 pM) }\end{array}$ & $8 C$ & $\begin{array}{l}\text { library } 12 \\
\text { (5.5 pM) }\end{array}$ \\
\hline
\end{tabular}

Libraries $1-6=$ mild negative energy balance animals. Libraries $7-12=$ severe negative energy balance animals. Libraries on the first flowcell were run at different concentrations (4.5-6.5 pM) to determine which concentration yielded optimum cluster yields. 
removal of reads that either failed to align to BCM4 or aligned to more than one position in the genome, and the removal of all but one of the reads that mapped to exactly the same position on the genome (i.e. putative PCR duplicates), an average of 3.8 million reads per lane were retained for read abundance calculation. An overview of these data is given in Additional file 1 and they have been deposited in NCBI's Gene Expression Omnibus and are accessible through GEO Series accession number GSE37544 (http://www.ncbi.nlm.nih.gov/geo/ query/acc.cgi?acc $=$ GSE37544).

\section{Transcriptional profile of cattle liver}

The lowest limit of detection was set to 5 or more uniquely aligned reads in at least one animal in either the SNEB or MNEB group. At this limit, 12,523 and 12,833 genes were detected as expressed in MNEB and SNEB animals respectively. This is similar to the number of human Ensembl genes detected in human $(12,191)$ and mouse $(11,201)$ liver tissue by polyA RNA-seq [25]. RNA-seq analysis of mouse and human liver by other groups showed that the 10 most highly expressed genes make up $20-40 \%$ of the mRNA pool whereas other tissue transcriptomes e.g. brain, kidney and testis are more complex with the 10 most highly expressed genes contributing to just $5-10 \%$ of the total mRNA [25]. We found that in the liver of cows in NEB, on average $16 \%$ of the total RNA-seq reads aligned to only 10 genes and $30 \%$ of the RNA-seq reads mapped to just 619 genes (Figure 1A) per lane. However, when we normalised for gene length by calculating values for fragments per kilobase of exon per million fragments mapped (FPKM) to get a better indication of relative transcript number, the top 10 most highly expressed genes represented only $0.77 \%$ of the transcript pool. There were highly abundant transcripts (FPKM >50) for approximately 1,200 genes in hepatic tissue analysed in the current study (Figure 1B). Depletion of very highly expressed long transcripts may be necessary to increase the sequencing coverage of less abundant shorter transcripts in mammalian liver RNA-seq libraries.

\section{Lane effect}

To further determine whether the differential expression observed between MNEB and SNEB was due to a real difference between the groups and not simply random variation caused by running samples on different lanes (i.e. lane effect), correlation analysis was performed between FPKM values from the two treatment groups and also the same 9 libraries run as technical replicates on two different lanes (Figure 2). This showed that the difference in FPKM values $\left(r^{2}=0.9783\right.$ NEB vs. SNEB $r^{2}=0.9905$ Lane A vs. Lane B) was predominately due to effect of treatment and not lane effect.
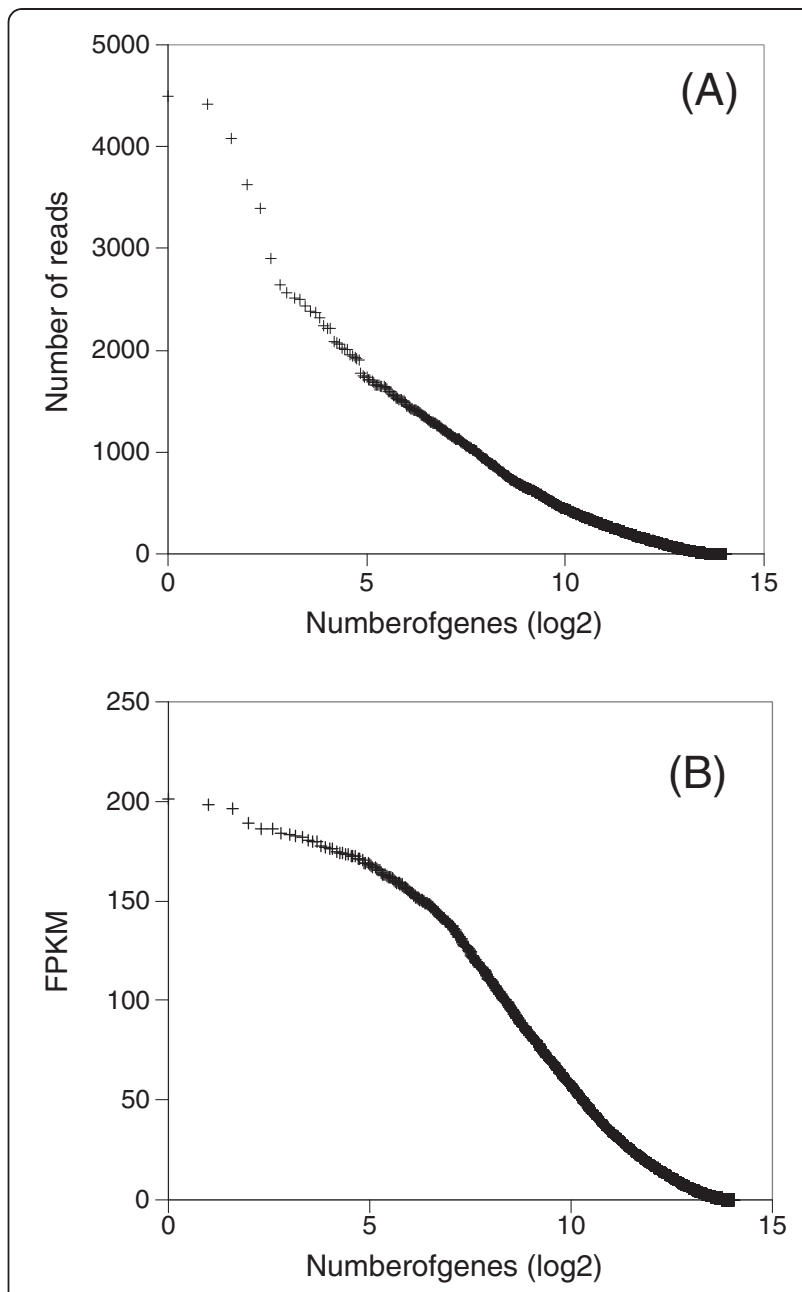

Figure 1 Complexity of NEB cattle liver transcriptome. (A) Average number of reads across 21 flowcell lanes aligning to genes. (B) Average FPKM values for genes across 21 flowcell lanes.

\section{Identification of SDE genes}

DEseq is an R-based software package that was developed specifically for the identification of SDE genes from raw counts of sequence reads generated by RNA-seq analysis that uniquely align to genes. RNA-seq library 1 (corresponding to MNEB animal 1) was not included in these analyses as this animal subsequently became ill and a possible underlying infection may have skewed the gene expression data generated. DEseq analysis showed that 413 genes were SDE (FDR of 0.1\%), 201 of which had a fold change of $\geq 2$ (either up or down) between MNEB and SNEB animals (Additional file 2).

\section{Comparison of microarray and RNA-seq}

We previously analysed the same MNEB and SNEB liver RNA samples using the Affymetrix $23 \mathrm{~K}$ bovine gene microarray platform and, of the 5,229 genes which were detected as expressed, 416 were identified as SDE by the puma method using the recommended cut-off P-like 

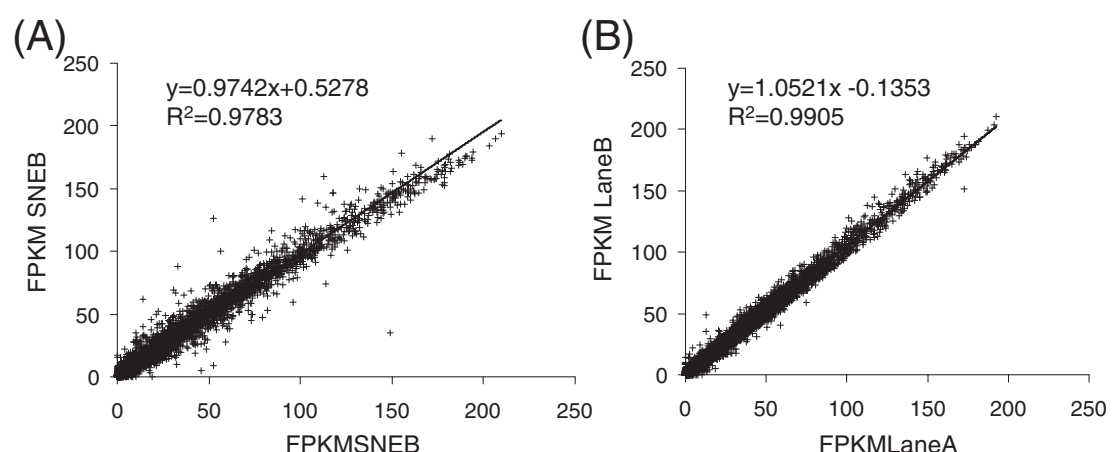

Figure 2 Lane effect. (A) Average FPKM values for each gene in SNEB animals correlated with average FPKM values for each gene in MNEB animals. (B) Average FPKM values for each gene for libraries 1, 2, 5, 6, 7, 10, 11, 12 run on lanes 1A, 3B, 3A, 2B, 4B, 7A, 7B, 1B respectively correlated with average FPKM values for each gene for libraries 1, 2, 5, 6, 7, 10, 11, 12 run on lanes 2A, 1C, 4A, 3C, 4C, 8A, 5A, 7B, 8C respectively.

value of $P<0.05$ [11]. Of these, only 56 had fold changes of $\geq 2$ (up or down) in SNEB animals. 55 of the 416 microarray and 413 RNA-seq SDE genes, and 27 of the 201 RNA-seq and 56 microarray $\geq 2$-fold SDE genes, were detected as SDE by both platforms (Additional file 3). All genes detected as SDE on both platforms had fold changes in the same direction. This approximate comparison of the two data sets shows that, particularly for the lower fold changes, many genes were detected as SDE on one platform but not the other. However, RNAseq detected expression of more than twice as many genes as the $23 \mathrm{~K}$ bovine microarray, and nearly four times as many $\geq 2$-fold SDE genes.

\section{Physiology of SNEB model}

Soon after calving in high-yielding dairy cows, nutritional and energetic demands can increase 4-fold within a single day as the animal undergoes a physiological upheaval to meet the demand by the mammary gland for substrates, particularly glucose, for milk production. The main changes of this upheaval are a reduction of insulin concentration in the blood, a reduction of lipogenesis in adipocytes, an increase in export of fats from adipose to liver, and a dramatic increase in gluconeogenesis in liver. Unlike non-ruminants, where glucose is absorbed via the small intestine, approximately $90 \%$ of all glucose in ruminants is synthesised by gluconeogenesis in the liver from volatile fatty acids (VFAs) derived from bacterial fermentation in the rumen. VFAs are absorbed across the rumen wall and into the hepatic portal vein which delivers them to the liver. The increase in hepatic gluconeogeneis at the onset of lactation leads to production of very high quantities of glucose. The glucose entry into the blood stream in top performing cows producing $90 \mathrm{~kg}$ milk per day has been estimated at $7.4 \mathrm{~kg}$ per day of which $4.4 \mathrm{~kg}$ will end up as lactose in milk [26].

In our model we were comparing two groups of lactating animals in the early post-calving period (13 or
14 days post-calving). One of these groups was in MNEB and the other was in a state of SNEB which was artificially induced by restricting feed and trebling the milking frequency. From this we hoped to identify novel genes and pathways which are important in SNEB in high-yielding dairy cows. Hormones, metabolites and aspects of liver composition measured at the time of tissue collection showed that, as predicted, relative to MNEB cows, SNEB cows had undergone significant $(P<0.05)$ changes in energy balance $(-3.6$-fold $)$, blood glucose ( -1.5 -fold), blood NEFA (+ 4.7-fold), blood BHB (+7.4), blood IGF1 (-4.8-fold), liver TAG (+3.9-fold), liver glycogen (-10.4-fold). Blood insulin, oestradiol, urea and liver weight were not different between SNEB and MNEB animals [9]. The level of cholesterol was also lower in the SNEB cows (Additional file 4). These changes are widely known to occur in SNEB cows [10] where there is an increase in the export of NEFAs from adipose to the liver which exceeds the normal oxidative capacity of the liver. This results in an increase in ketone bodies (BHB) in the blood resulting from partially oxidised NEFAs, and an accumulation of TAGs in the liver. In addition, IGF1 levels drop as hepatocytes become refractory to growth hormone (GH). Under normal conditions, growth hormone $(\mathrm{GH})$ is released from the anterior pituitary in the brain, binds to the growth hormone receptor (GHR) on the surface of hepatocytes, and this binding triggers a signalling cascade that leads to increased IGF1 synthesis by the liver for export to the blood. In NEB cows the liver becomes refractory to GH and this results in low circulating IGF1 [10].

\section{Over-represented KEGG pathways}

In our RNA-seq study, nine KEGG pathways were associated with $\geq 2$-fold SDE genes most of these were related to metabolism of fats (Table 2, Figures 3, 4, Additional file 5). Pathways related to the metabolism of carbohydrates were only over-represented when SDE genes 
Table 2 Summary of KEGG pathways associated with $\geq 2$ fold SDE genes

\begin{tabular}{lll}
\hline $\begin{array}{l}\text { Pathway } \\
\text { code }\end{array}$ & Pathway name & $\begin{array}{l}\text { Over represented } \\
\boldsymbol{P} \text { value }\end{array}$ \\
\hline 00140 & Steroid hormone biosynthesis & 0.000163 \\
03320 & PPAR signalling pathway & 0.000903 \\
04976 & Bile secretion & 0.000960 \\
04977 & Vitamin digestion and & 0.003082 \\
04975 & absorption & \\
02010 & Fat digestion and absorption & 0.004934 \\
00592 & ABC transporters & 0.006003 \\
01040 & a-linolenic acid metabolism & 0.006639 \\
00830 & Biosynthesis of unsaturated & 0.022054 \\
\hline
\end{tabular}

Pathway maps for KEGG pathways associated with $\geq 2$-fold SDE genes (FDR0.1\%) that are not included in the main text are in Additional file 5.

with $<2$-fold change were included in the pathway analysis, (Table 3, Additional file 6). This suggests that in our model, at the level of transcription in the liver, to compensate for the reduced supply of VFAs from the liver due to feed restriction, the major changes in hepatic gene expression in SNEB cows were related to the alternative supply of carbon from fat for gluconeogenesis. The most interesting finding from our RNA-seq analysis was that 'Steroid hormone biosynthesis', a process which is normally exclusive to the gonads and adrenal glands, was the KEGG pathway most significantly associated with $\geq 2$-fold SDE genes in SNEB cow liver. Details of this and other selected pathways are discussed below in the context of the expected physiological changes in the liver of SNEB cows.

\section{Carbohydrate metabolism}

The 1.5-fold drop in blood glucose in SNEB cows would suggest decreased gluconeogenesis in the liver of these animals. However, only a minor decrease $(\leq 2$-fold) of pyruvate carboxylase (PYK (KEGG pathway map) = PKLR (SDE gene list), one of the major rate limiting enzymes in gluconeogenesis [27] was identified in the KEGG 'Insulin signalling pathway' (Additional files 2 and 6). The KEGG pathway 'Glycolysis/gluconeogeneis' was not identified as over-represented. This indicates that, in feed-restricted SNEB animals, there was only a slight decrease in the rate of gluoneogenesis and that demands for carbon for this process were mostly being met by oxidation of NEFAs transported from adipose. There were no gene pathways to related to the 10-fold drop in liver glycogen in observed in SNEB cows even though genes coding for enzymes involved in glycogen metabolism (e.g. PYG, PHK) were detected as expressed in the KEGG 'Insulin signalling pathway' (Additional file 6).
Major changes in transcription of genes controlling glycogenolysis may have occurred prior to collection of liver tissue for RNA extraction.

\section{Uncoupling of the GH/IGF1 axis}

Although pathways related to the GH/IGF1 axis were not identified as overrepresented among the SDE genes identified by RNA-seq, several genes within in this pathway were detected as SDE (GHR -1.6-fold, IGFALS -5fold, IGF1 -5-fold) (Additional file 2). The KEGG 'p53 signalling pathway', which is related to stress and includes IGF1, was overrepresented (Table 3). Hepatic expression of IGF1, GHR and IGFALS is well known to be reduced in SNEB cows and has been previously described $[9,11]$. Interestingly, for the same total RNA samples, IGF1 was detected as SDE by both RNA-seq and qPCR but not by microarray (Additional file 3) [11], even though the amount of blood IGF1, which is made mainly in the liver, was significantly reduced in SNEB cows. Microarray also failed to detect GHR as SDE. However, our microarray study did, whereas our RNAseq study did not, identify IGFBP2 and IGFBP3 (genes encoding IGF1 binding proteins) as $\operatorname{SDE}(\leq 2$-fold) in SNEB cow liver.

\section{TAG accumulation and increased blood NEFA}

Increased TAG accumulation in the liver was reflected by overrepresentation of the KEGG 'PPAR signalling pathway' (Table 2, Figure 3) as angiopoietin-like 4 gene (PGAR $($ KEGG pathway map, Figure 3$)=$ ANGPTL4 $($ SDE gene list, Additional file 2) was upregulated in this pathway. Angiopoietin-like protein 4 has emerged as a key regulator of plasma cholesterol, triglyceride, and NEFA concentrations and, in cattle, is most highly expressed in the liver and adipose [28]. The upregulation of ANGPTL4 due to fasting, corresponding to the SNEB condition, was also reported in adipose tissues of lactating goats [29]. Upregulation of ANGPTL4, which is stimulated by PPAR agonists, leads to inhibition of lipoprotein lipase activity in adipose, reduced VLDL-TAG utilisation, and increased lipolysis. This gene was previously identified by microarray to be up-regulated in the liver of induced ketosis cows [10] and SNEB cows [11] and it was proposed that increased ANGPTL4 serves as a signal for lipolysis and contributes to sustained release of NEFA and lipid accumulation in the liver [10].

\section{Oxidation of NEFAs and ketogenesis}

Due to the higher amount of NEFAs in the blood of SNEB animals, we were expecting changes in expression of genes to reflect increased oxidation of fatty acids in SNEB animals. However, although eight of the nine fatty acid oxidation genes in the KEGG PPAR signalling pathway were detected, none were SDE (Additional file 2). 


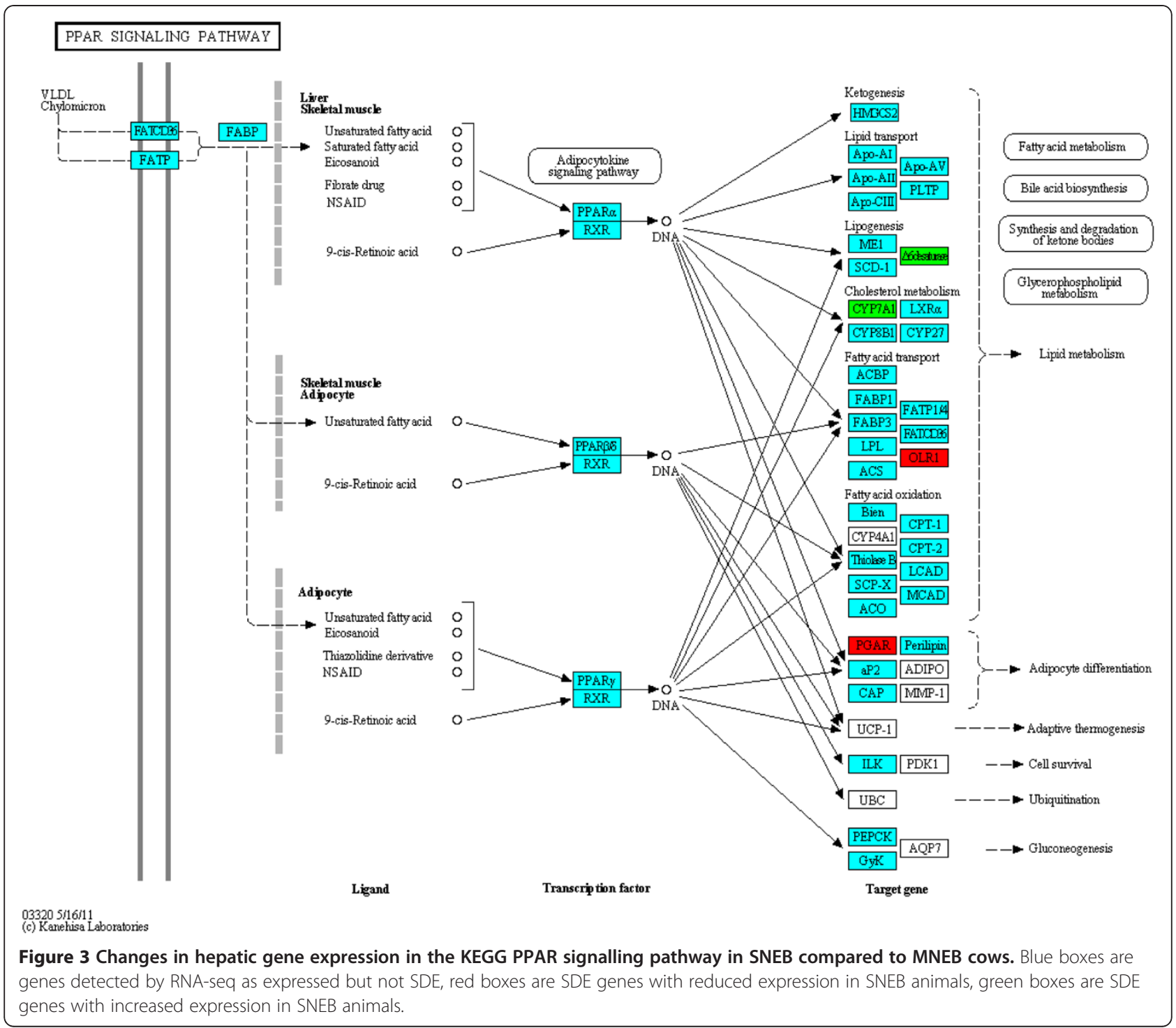

Acetyl-CoA carboxylase beta $(A C A C B)$ which is thought to control fatty acid oxidation was SDE (upregulated 3fold) in RNA-seq but not picked up in the pathway analysis. KEGG PPAR signalling pathway is also involved in ketogenesis but, although the main gene involved in this (HMGCS2) was detected, it was not identified as SDE. This may be because at the time of slaughter there was little difference in the rate of oxidation and ketogenesis between the two groups, or that there were differences but not at the level of transcription. OLR1 (oxidized low density lipoprotein (lectin-like) receptor 1) is included in the KEGG PPAR signalling pathway and was detected as upregulated (although read counts were very low for this gene). The protein encoded by OLR1 binds, internalizes and degrades oxidized low-density lipoprotein (oxLDL) and is thought to have a role in lowering systemic oxidative stress by removal of oxLDL from blood.

\section{Impaired PUFA synthesis}

The most SDE gene we identified by RNA-seq was FADS2 (previously named $\Delta 6$ desaturase) which was down-regulated in SNEB animals (Figure 3, Additional files 2) and featured in 3 of the nine $\geq 2$-fold KEGG pathways ( $\alpha$-Linolenic acid metabolism, Biosynthesis of unsaturated fatty acids, and PPAR signalling) (Additional file 5). FADS1 was also shown to be down-regulated in SNEB animals (Additional files 2). FADS1 and FADS2 were previously identified by microarray as downregulated in liver of cows with induced clinical ketosis (days 9-14 post partum) [10]. However, in our microarray study, FADS2, but not FADS1, was identified as SDE. FADS2 catalyses the initial desaturation step of $\alpha$ - 


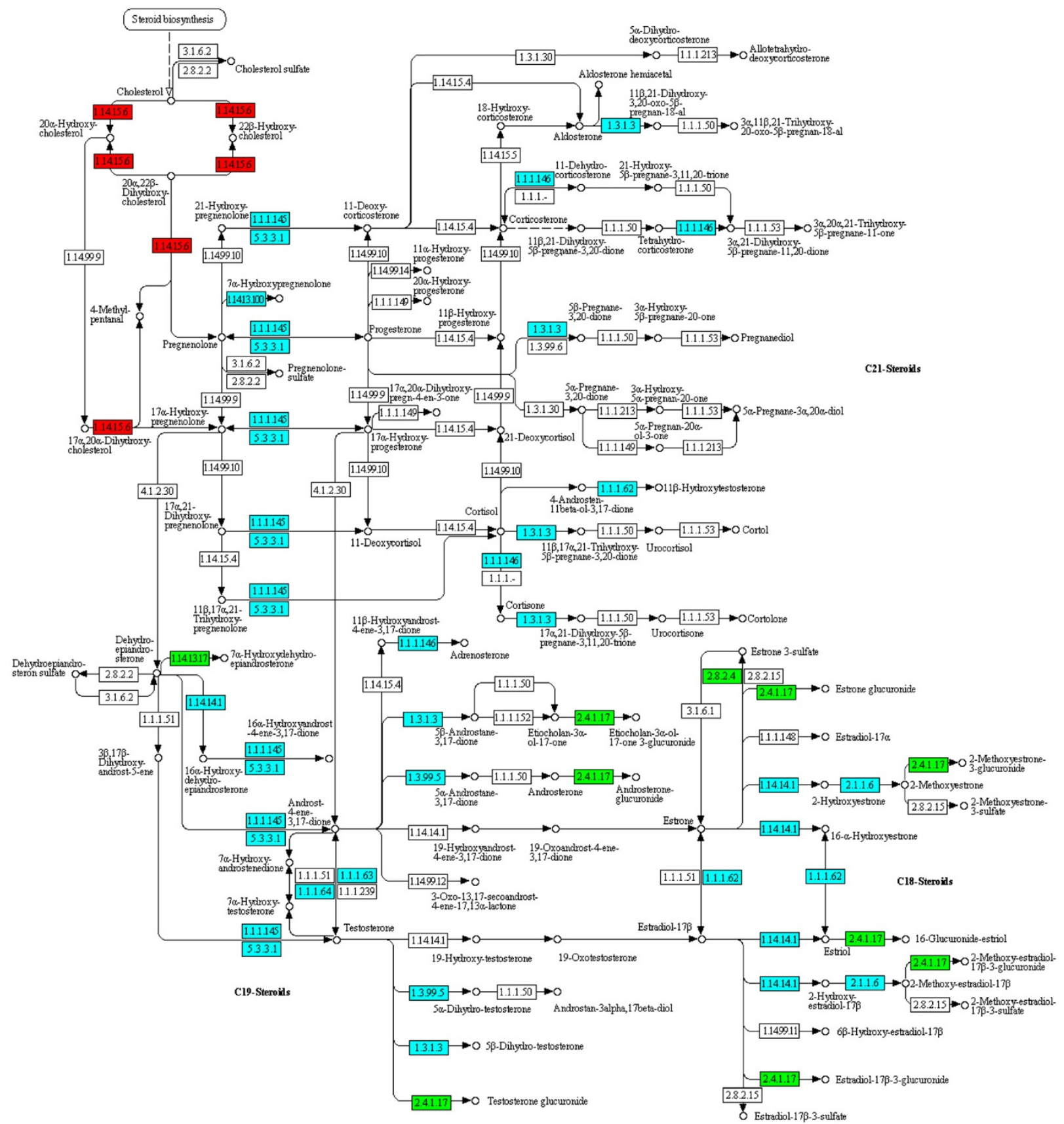

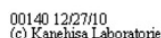

Figure 4 Changes in hepatic gene expression in the KEGG Steroid hormone biosynthesis pathway in SNEB compared to MNEB cows. Blue boxes are KEGG enzymes coded for by genes detected by RNA-seq as expressed but not SDE, red boxes are KEGG enzymes coded for by SDE genes with reduced expression in SNEB animals, green boxes are KEGG enzymes coded for by SDE genes with increased expression in SNEB animals. KEGG enzyme 1.14.15.6 is coded for by HSA CYP11A1, KEGG enzyme 1.14.13.17 is coded for by HSA CYP7A1, KEGG enzyme 2.4.1.17 is coded for by HSA UG2A1, KEGG enzyme 2.8.2.4 is coded for by HSA SULT1E1. 
Table 3 Summary of KEGG pathways associated with all SDE genes

\begin{tabular}{lll}
\hline $\begin{array}{l}\text { Pathway } \\
\text { code }\end{array}$ & Pathway name & $\begin{array}{l}\text { Over represented } \\
\boldsymbol{P} \text { value }\end{array}$ \\
\hline 04976 & Bile secretion & 0.001483 \\
00140 & Steroid hormone biosynthesis & 0.002046 \\
04950 & Maturity onset diabetes & 0.004947 \\
00910 & of the young & \\
00061 & Nitrogen metabolism & 0.007765 \\
05219 & Fatty acid biosynthesis & 0.011248 \\
03320 & PPAR signalling pathway & 0.018112 \\
04910 & Bladder cancer & 0.018941 \\
04115 & p53 signalling pathway & 0.020756 \\
04977 & Insulin signalling pathway & 0.021653 \\
00592 & Vitamin digestion and absorption & 0.026059 \\
04975 & a-linolenic acid metabolism & 0.030405 \\
04080 & Fat digestion and absorption & 0.034363 \\
& Neuroactive ligand-receptor & 0.040178 \\
\hline
\end{tabular}

Pathway maps for KEGG pathways associated with all SDE genes (FDR 0.1.\%) are in Additional file 6.

linolenic acid (C18:3) and linoleic acid (C18:2) in the enzyme cascade that ultimately leads, via $F A D S 1$, to synthesis of the long chain polyunsaturated fatty acids (LC-PUFAs) arachidonic acid (C20:4) and eicosapentaenoic acid (C20:5). Synthesis of LC-PUFAs arachidonic acid and eicosapentaenoic acid mainly occurs in the liver and adipose [30] and suppression of their synthesis in SNEB cows may contribute to the poor fertility in high-yielding dairy herds. It was recently reported that polymorphisms in FADS1 and FADS2 were associated with reduced serum arachidonic levels in humans [31]. Also, deletion of FADS2 gene expression in mice leads to sterility in both male and female mice without affecting their viability [32].

\section{Unexpected overrepresentation of KEGG pathway 'steroid hormone biosynthesis'}

One of the most interesting findings of our RNA-seq study was that the KEGG pathway 'Steroid hormone biosynthesis' (Table 2, Figure 4) showed the most significant association with $\geq 2$-fold SDE genes in SNEB animals. This is unexpected as, under normal conditions, steroid hormone biosynthesis occurs almost exclusively in the adrenal glands and gonads, whereas liver is the site for steroid hormone inactivation. Four genes were changed in this pathway. CYP11A1 was up-regulated while UGT2a1, SULTE1 and CYP7A1 were down-regulated. CYP11A1 was also identified as SDE in SNEB cow liver in our microarray study [11] but not in the liver of induced ketosis cows [10]. CYP7A1, UGT2A1 and SULT1E1 were not identified as SDE in either of those studies. CYP7A1 catalyses the rate limiting step of conversion of cholesterol to bile acids and was also included in the KEGG pathway 'Bile secretion' which was overrepresented in the $\geq 2$-fold SDE genes identified by RNAseq (Table 2, Additional file 5). UGT2A1 and SULT1E1 inactivate oestrogens by glucuronidation and sulphation respectively, prior to their excretion. The down-regulation of these genes in the liver of SNEB animals indicates that bile synthesis and inactivation of oestrogens in liver is reduced in SNEB possibly to conserve cholesterol which was reduced in SNEB animals (Additional file 4). This also suggests that lower blood concentration of oestrodiol in preovulatory high yielding dairy cows may not be due to increased oestrogen inactivation in liver.

\section{CYP11A1, a novel gene in metabolic adaptations to post- partum bovine SNEB}

Although we detected upregulation of CYP11A1 in liver of SNEB cows in our microarray study, 'Steroid hormone biosynthesis' was not associated with the microarray SDE genes, probably because CYP7A1, UGT2A1 and SULTE1 were not identified as SDE, so the implications of upregulation of CYP11A1 in SNEB cows were not discussed [11]. To the knowledge of the authors, other than in our own microarray study, expression of CYP11A1 has not been previously described in adult cow liver. Other workers found CYP11A1 to be undetectable by qPCR in the liver of adult cows and consequently used liver as a negative control for expression studies of CYP11A1 in granulosa [33]. However, CYP11A1 does appear to be expressed in foetal and juvenile cows [34]. Up-regulation of this gene is unexpected as it catalyses the initial step of conversion of cholesterol into pregnenolone which is then converted by CYP17A1 to dehydroepiandrosterone (DHEA). DHEA is the precursor for a number of steroid hormones including oestrogen. Although other tissues, such as brain and foetal rat liver, also express the enzymes for steroid biosynthesis [35] these enzymes are not normally expressed in adult mammalian liver. Unexpected up-regulation of CYP11A1 was also observed in vitro in human hepatocytes after treatment with adenovirus expressing PGC-1 alpha [35] which is a key integrator of many of the signalling pathways that are induced in the liver and muscle upon fasting. In that study, CYP17A1 was also upregulated and the authors proposed that DHEA (or one of its metabolites) in liver could be involved in a novel hepatic signalling pathway or may serve to protect hepatocytes from the increased oxidative state brought on by fasting. DHEA has been shown to protect endothelial cells from apoptosis. They also suggested that CYP11A1 expression in feed restricted hepatocytes may be involved in regulation of cholesterol homeostasis as CYP11A1 catalyses modifications on cholesterol to produce oxysterols 
which are known ligands for liver X receptors (LXRs). LXRs regulate cholesterol homeostasis and lipid metabolism. It would therefore be interesting to see if DHEA levels in SNEB cow liver are elevated. In both our microarray and RNA-seq study, SDE genes included in the IPA canonical signalling pathway ' $L X R / R X R$ activation' were changed (ABCG8, ACACA (downregulated); $A R G$, CCL2, IL1RN, RXRG (upregulated)). In our RNA-seq data, many of the genes, including CYP17A1, in the KEGG pathway 'Steroid hormone biosynthesis' were not detected as expressed (Additional file 5). This may either be due to the fact that these genes are duplicated in the genome and thus reads were eliminated because they mapped to more than one locus (e.g. CYP17A1 (human gene name) has an exact copy (LOC784256) at another position in the Bos taurus genome), transcript abundance was too low for detection, or that they are not expressed in the liver thus preventing complete synthesis of steroid hormones in this organ.

\section{Conclusions}

RNA-seq analysis showed that the major changes at the level of transcription in the liver of SNEB cows were related to fat metabolism. Unexpectedly, one of the most significantly changed biological pathways was 'Steroid hormone biosynthesis' where CYP11A1 was upregulated. In adult cows, this gene is normally only expressed in the adrenal glands and ovaries and not liver. CYP11A1 is a potentially novel gene that may play a key role in metabolic adaptations to NEB in high-yielding dairy cows.

\section{Materials and methods}

Animal model

The animal model employed in this study has been described previously [9], and all procedures were carried out under license in accordance with the European Community Directive 86-609-EC. The nutritional and lactational management regime employed were designed to create significant divergence in the energy balance (EB) profiles of the cows in early lactation. In brief, multiparous Holstein-Friesian cows $(n=24)$ were blocked 2 wk prior to expected calving date according to parity, body condition score (BCS), and previous lactation yield (average lactation $6,477 \pm 354 \mathrm{~kg}$ ) and randomly allocated to mild (MNEB, $\mathrm{n}=12$ ) or severe $(\mathrm{SNEB}, \mathrm{n}=12$ ) NEB groups. MNEB cows were fed ad libitum grass silage and $8 \mathrm{~kg} /$ day concentrates and milked once daily; SNEB cows were fed $25 \mathrm{~kg} /$ day silage and $4 \mathrm{~kg} /$ day concentrate and milked three times daily. Measurements of BCS and EB were used to select cows that showed extremes in EB from each group (MNEB, $n=5$; SNEB, $n=6$ ). Cows were slaughtered on days 6-7 of the first follicular wave after calving (mean number of days postpartum: MNEB mean $13.6 \pm 0.75$, range $11-15$; SNEB mean $14.3 \pm 0.56$, range $13-16$ ), based on daily transrectal ultrasonography.

\section{Collection of liver tissue for RNA and TAG analysis}

The entire liver was removed within 15-30 min after slaughter and weighed. Samples weighing approximately $1 \mathrm{~g}$ were dissected, rinsed in RNase-free phosphate buffer, snap-frozen in liquid nitrogen, and stored at $-80^{\circ} \mathrm{C}$. For triacylglyceride (TAG) analysis, total lipids were extracted from $50 \mathrm{mg}$ samples of liver as previously described [9].

\section{Blood sampling and metabolite assays}

Stabilized (EDTA-treated) whole blood samples were collected on the day of slaughter by jugular venipuncture for haematological analysis. Blood samples were analyzed for glucose, NEFA, $\beta$-hydroxybutyrates (BHB), and urea using appropriate kits and an ABX Mira autoanalyser (ABX Mira, Cedex, France). All metabolite assay coefficients of variation were low and typically $<5 \%$.

\section{RNA extraction and quality analysis}

Total RNA was prepared from 100-200 mg of fragmented frozen liver tissue using the TRIzol reagent (SigmaAldrich, Dorset, UK). Tissue samples were homogenized in $3 \mathrm{ml}$ of TRIzol reagent and chloroform and subsequently precipitated using isopropanol (Sigma Chemical, Wicklow, Ireland). RNA samples were stored at $-80^{\circ} \mathrm{C}$. $20 \mu \mathrm{g}$ of total RNA from each sample for genomic DNA contamination was treated with the RNase-free DNase set (QIAGEN, Crawley, West Sussex, UK) and purified it using the RNeasy mini kit in accordance with guidelines supplied (QIAGEN, Crawley, West Sussex, UK). RNA quality and quantity were assessed using automated capillary gel electrophoresis on a Bioanalyzer 2100 with RNA 6000 Nano Labchips according to manufacturer's instructions (Agilent Technologies Ireland, Dublin, Ireland). Samples of RNA had $28 \mathrm{~S} / 18 \mathrm{~S}$ ratios ranging from 1.8 to 2.0 and RNA integrity number values of between 8.0 and 10.0.

\section{Preparation and sequencing of polyA mRNA-seq ilumina libraries}

polyA RNA was isolated from 5-10 ug total RNA with oligo(dT) beads using two rounds of oligo-dT purification. 5-10 ug RNA was fragmented with zinc fragmentase (Applied Biosystems, Warrington, UK), first strand cDNA synthesis was performed using the Invitrogen random hexamer primers and SuperScript II (Invitrogen), second strand synthesis was performed using Invitrogen DNA Polymerase 1 (Invitrogen). End repair and polyadenylation were performed using NEB Next Tailing Module (New England Biolabs). ${ }^{\circledR}$ End Repair Module 
and NEB Next dA- Illumina single read adapters were ligated to blunt ended, polyadenylated fragments with a NEB Quick ligation kit (New England Biolabs). Adapterligated cDNA fragment libraries were run on an Illumina GAII using version 3 sequencing kits and version 3 single read cluster generation kits.

\section{Read alignment}

The reads from each of the lanes were aligned separately to the BCM4 genome assembly [23], using the ultrafast short read aligner, Bowtie (version 0.12.3) [24]. Fastq output files from the sequencer were used as input, specifying the following options: quality scores are ASCII characters equal to the Phred quality scores plus 64 (-Solexa1.3-quals); the maximum number of mismatches allowed in the first 28 bases is $2(-\mathrm{n} 2,-128)$; suppressing all alignments for any read that has more then 1 reportable alignment $(-\mathrm{m} 1)$; retained alignments were reported in SAM format $(-S)$.

\section{Read abundance calculation}

The abundance of mRNAs for all annotated genes from the ENSEMBL v59 annotation of the bovine genome [36], was calculated using the software package HTseq (version 0.4.4p6) (http://sourceforge.net/projects/htseq/). The script HTseq-count provided with this package was used to count the number of reads that mapped to each annotated gene, allowing, in some cases, for reads to partially overlap with the exons and still be counted for that gene (i.e. $-\mathrm{m}$ union). As our sequencing analysis included technical replicates of individual samples, we summed the counts for all these lanes, resulting in a single count for each gene for each sample. The counts for all the samples were collated into one file and any gene with fewer than 5 reads in all samples was excluded from the subsequent statistical analysis of differential gene expression.

\section{Identification of SDE genes and pathway analysis}

We identified SDE using DEseq (version 1.1.11) [21]. DEseq uses a generalisation of the Poisson model, the negative binomial distribution, to model biological and technical variance and test for differential expression between two experimental conditions. As there are nearly 30,000 genes annotated in the bovine genome, the statistical tests in each analysis were corrected for multiple testing using the Benjamini and Hochberg (BH) method [37] as implemented in R (version 2.12.0). All genes that were found to be SDE between the two experimental conditions (at an FDR p-value cut-off of 0.1) were retained for further analysis.

GOseq and KEGG pathway analysis tools were used to identify biological pathways that were significantly enriched in the data set of SDE genes (Table 2,
Additional file 3). To facilitate this, reads were firstly converted to their human orthologs. GOseq specifically adjusts for the higher abundance of reads from long or highly expressed genes in RNA-seq experiments when assessing a gene list for over-representation of biological functions [22]. Genes were mapped to the KEGG database [38] for pathway analysis using GOseq [22].

\section{Additional file}

\section{Additional file 1. Table of number and assignment of reads for each flowcell lane. \\ Additional file 2. Table SDE genes and their fold-change. \\ Additional file 3. Comparison of microarray and RNA-seq SDE genes. \\ Additional file 4. Graph of effect of SNEB compared to MNEB on blood concentrations of major NEB associated metabolites 1- 14 days post-calving. \\ Additional file 5 . Maps of KEGG pathways (associated with $\geq \mathbf{2}$ fold (FDR 0.1\%) SDE genes) overrepresented in SNEB animals. \\ Additional file 6. Maps of KEGG pathways associated with all SDE (FDR 0.1\%) genes.}

\section{Competing interests}

The authors declare that they have no competing interests.

\section{Acknowledgements}

This work was funded by Teagasc under the Irish National Development Plan and Chris Creevey is funded under the Science Foundation Ireland (SFI) Stokes lecturer scheme (07/SK/B1236A). The authors thank Alison Murphy and Amanda Lohan for their technical support and advice.

\section{Author details}

${ }^{1}$ Animal and Bioscience Research Department, Animal and Grassland Research and Innovation Centre, Teagasc, Grange, Dunsany, Co. Meath, Ireland. ${ }^{2}$ Animal and Bioscience Research Department, Animal and Grassland Research and Innovation Centre, Teagasc, Mellows Campus, Athenry, Co. Galway, Ireland.

\section{Authors' contributions}

MSM generated the RNA-seq libraries, co-ordinated the sequencing runs, helped with retrieval and analysis of data, carried out detailed interpretation of the pathway analysis, and prepared the main manuscript. SW extracted the RNA from NEB liver samples, conceived the idea of performing RNA-seq on the NEB RNA samples and contributed to the biological interpretation of the data and manuscript preparation. DL contributed to the bioinformatics of differential gene expression analysis and pathway analysis and writing and editing of the manuscript. DK, and DM co-ordinated tissue collection and RNA extraction from the NEB model, conducted metabolite studies on the model and contributed to the biological interpretation of the data and writing and editing of the manuscript. CC set up and performed the majority of the bioinformatics on the sequencing runs including data retrieval, read alignments, differential gene expression analysis, KEGG pathway analysis, data interpretation and co-wrote the main manuscript with MSM. All authors read and approved the final manuscript.

Received: 20 September 2011 Accepted: 07 May 2012 Published: 20 May 2012

\section{References}

1. Wathes DC, Cheng ZR, Chowdhury W, Fenwick MA, Fitzpatrick R, Morris DG, Patton J, Murphy JJ: Negative energy balance alters global gene expression and immune responses in the uterus of postpartum dairy cows. Physiol Genomics 2009, 39(1):1-13. 
2. Drackley JK: ADSA Foundation Scholar Award. Biology of dairy cows during the transition period: the final frontier? J Dairy Sci 1999, 82 (11):2259-2273.

3. Pullen DL, Liesman JS, Emery RS: A species comparison of liver slice synthesis and secretion of triacylglycerol from nonesterified fatty-acids in media. J Anim Sci 1990, 68(5):1395-1399.

4. Reynolds CK, Aikman PC, Lupoli B, Humphries DJ, Beever DE: Splanchnic metabolism of dairy cows during the transition from late gestation through early lactation. Journal of Dairy Science 2003, 86(4):1201-1217.

5. Morris DG, Waters SM, McCarthy SD, Patton J, Earley B, Fitzpatrick R, Murphy $J$ J, Diskin MG, Kenny DA, Brass A, et al: Pleiotropic effects of negative energy balance in the postpartum dairy cow on splenic gene expression: repercussions for innate and adaptive immunity. Physiol Genomics 2009, 39(1):28-37.

6. Pickett MM, Piepenbrink MS, Overton TR: Effects of propylene glycol or fat drench on plasma metabolites, liver composition, and production of dairy cows during the periparturient period. J Dairy Sci 2003, 86(6):21132121.

7. Wathes DC, Fenwick M, Cheng Z, Bourne N, Llewellyn S, Morris DG, Kenny $D$, Murphy J, Fitzpatrick R: Influence of negative energy balance on cyclicity and fertility in the high producing dairy cow. Theriogenology 2007, 68:s232-s241.

8. Loor JJ: Genomics of metabolic adaptations in the peripartal cow. Animal 2010, 4(7):1110-1139.

9. Fenwick MA, Fitzpatrick R, Kenny DA, Diskin MG, Patton J, Murphy JJ, Wathes DC: Interrelationships between negative energy balance (NEB) and IGF regulation in liver of lactating dairy cows. Domest Anim Endocrinol 2008, 34(1):31-44.

10. Loor JJ, Everts RE, Bionaz M, Dann HM, Morin DE, Oliveira R, Rodriguez-Zas SL, Drackley JK, Lewin HA: Nutrition-induced ketosis alters metabolic and signalling gene networks in liver of periparturient dairy cows. Physiol. Genomics 2007, 32:105-116.

11. McCarthy SD, Waters SM, Kenny DA, Diskin MG, Fitzpatrick R, Patton J, Wathes DC, Morris DG: Negative energy balance and hepatic gene expression patterns in high-yielding dairy cows during the early postpartum period: a global approach. Physiol Genomics 2010, 42A(3):188199.

12. Huang $\mathrm{W}$, Khatib $\mathrm{H}$ : Comparison of transcriptomic landscapes of bovine embryos using RNA-Seq. BMC Genomics 2010, 11:711.

13. Mortazavi A, Williams BA, McCue K, Schaeffer L, Wold B: Mapping and quantifying mammalian transcriptomes by RNA-Seq. Nat Methods 2008, 5 (7):621-628.

14. Wang Z, Gerstein M, Snyder M: RNA-Seq: a revolutionary tool for transcriptomics. Nat Rev Genet 2009, 10(1):57-63.

15. Kim TK, Hemberg M, Gray JM, Costa AM, Bear DM, Wu J, Harmin DA Laptewicz M, Barbara-Haley K, Kuersten S, et al: Widespread transcription at neuronal activity-regulated enhancers. Nature 2010, 465(7295):182-187.

16. Sultan $M$, Schulz MH, Richard $H$, Magen A, Klingenhoff $A$, Scherf $M$, Seifert $M$, Borodina T, Soldatov A, Parkhomchuk D, et al: A global view of gene activity and alternative splicing by deep sequencing of the human transcriptome. Science 2008, 321(5891):956-960.

17. Cánovas A, Rincon G, Islas-Trejo A, Wickramasinghe S, Medrano JF: (2010) SNP discovery in the bovine milk transcriptome using RNA-Seq technology. Mamm Genome 2010, 21(11-12):592-598.

18. Maher CA, Palanisamy N, Brenner JC, Cao X, Kalyana-Sundaram S, Luo S, Khrebtukova I, Barrette TR, Grasso C, Yu J, et al: Chimeric transcript discovery by paired-end transcriptome sequencing. Proc Natl Acad Sci U S A 2009, 106(30):12353-12358.

19. Oshlack A, Robinson MD, Young MD: From RNA-seq reads to differential expression results. Genome Biol 2010, 11(12):220.

20. Garber M, Grabherr MG, Guttman M, Trapnell C: Computational methods for transcriptome annotation and quantification using RNA-seq. Nat Methods 2011, 8(6):469-477.

21. Anders S, Huber W: Differential expression analysis for sequence count data. Genome Biol 2010, 11(10):R106.

22. Young MD, Wakefield MJ, Smyth GK, Oshlack A: Gene ontology analysis for RNA-seq: accounting for selection bias. Genome Biol 2010, 11(2):R14.

23. Elsik CG, Tellam RL, Worley KC, Gibbs RA, Muzny DM, Weinstock GM, Adelson DL, Eichler EE, Elnitski L, Guigo R, et al: The genome sequence of taurine cattle: a window to ruminant biology and evolution. Science 2009, 324(5926):522-528.
24. Langmead B, Trapnell C, Pop M, Salzberg SL: Ultrafast and memoryefficient alignment of short DNA sequences to the human genome. Genome Biol 2009, 10(3):R25.

25. Ramskold D, Wang ET, Burge CB, Sandberg R: An abundance of ubiquitously expressed genes revealed by tissue transcriptome sequence data. PLoS Comput Biol 2009, 5(12):e1000598.

26. Aschenbach JR, Kristensen NB, Donkin SS, Hammon HM, Penner GB: Gluconeogenesis in dairy cows: the secret of making sweet milk from sour dough. lubmb Life 2010, 62(12):869-877.

27. Pershing RA, Moore SD, Dinges AC, Thatcher WW, Badinga L: Short communication: hepatic gene expression for gluconeogenic enzymes in lactating dairy cows treated with bovine somatotropin. Journal of Dairy Science 2002, 85(3):504-506.

28. Mamedova LK, Robbins K, Johnson BJ, Bradford BJ: Tissue expression of angiopoietin-like protein 4 in cattle. J Anim Sci 2010, 88(1):124-130.

29. Faulconnier Y, Chilliard Y, Montazer Torbati MB, Leroux C: The transcriptomic profiles of adipose tissues are modified by feed deprivation in lactating goats. Comparative Biochemistry and Physiology, Part D 2011, 6(2):139-149.

30. Jacobi SK, Lin X, Corl BA, Hess HA, Harrell RJ, Odle J: Dietary arachidonate differentially alters desaturase-elongase pathway flux and gene expression in liver and intestine of suckling pigs. J Nutr 2011, 141(4):548553.

31. Lattka E, Illig T, Koletzko B, Heinrich J: Genetic variants of the FADS1 FADS2 gene cluster as related to essential fatty acid metabolism. Curr Opin Lipidol 2010, 21(1):64-69.

32. Stoffel W, Holz B, Jenke B, Binczek E, Gunter RH, Kiss C, Karakesisoglou I, Thevis M, Weber AA, Arnhold S, et al: Delta6-desaturase (FADS2) deficiency unveils the role of omega3- and omega6-polyunsaturated fatty acids. EMBO J 2008, 27(17):2281-2292.

33. Vanselow J, Spitschak M, Nimz M, Furbass R: DNA methylation is not involved in preovulatory down-regulation of CYP11A1, HSD3B1, and CYP19A1 in bovine follicles but may have a role in permanent silencing of CYP19A1 in large granulosa lutein cells. Biol Reprod 2010, 82(2):289298

34. Bovine gene atlas http://bovine atlas.msstate.edu: Bovine gene atlas.: ; http:// bovine atlas.msstate.edu.

35. Grasfeder LL, Gaillard S, Hammes SR, Ilkayeva O, Newgard CB, Hochberg RB, Dwyer MA, Chang CY, McDonnell DP: Fasting-induced hepatic production of DHEA is regulated by PGC-1alpha, ERRalpha, and HNF4alpha. Mol Endocrinol 2009, 23(8):1171-1182.

36. Flicek P, Aken BL, Ballester B, Beal K, Bragin E, Brent S, Chen Y, Clapham P, Coates G, Fairley S, et al: Ensembl's 10th year. Nucleic Acids Res 2010, 38 : D557-D562.

37. Benjamini $Y$, Hochberg $Y$ : Controlling the false discovery rate - a practical and powerful approach to multiple testing. Journal of the Royal Statistical Society Series B-Methodological 1995, 57(1):289-300.

38. KEGG pathway data basehttp://www.genome.jp/kegg/pathway.html: KEGG pathway data base http://www.genome.jp/kegg/pathway.html.

\section{doi:10.1186/1471-2164-13-193}

Cite this article as: McCabe et al:: RNA-seq analysis of differential gene expression in liver from lactating dairy cows divergent in negative energy balance. BMC Genomics 2012 13:193.

\section{Submit your next manuscript to BioMed Central and take full advantage of:}

- Convenient online submission

- Thorough peer review

- No space constraints or color figure charges

- Immediate publication on acceptance

- Inclusion in PubMed, CAS, Scopus and Google Scholar

- Research which is freely available for redistribution 